\title{
Effect of low-dose diuretics on the level of serum cystatin $C$ and prognosis in patients with asymptomatic chronic heart failure
}

\author{
YUAN ZHANG, YANMING LI and GUANCHANG CHENG \\ Department of Cardiology, Henan University Huaihe Hospital, Kaifeng, Henan 475000, P.R. China
}

Received October 5, 2014; Accepted October 14, 2015

DOI: $10.3892 /$ etm.2015.2837

\begin{abstract}
The aim of the present study was to investigate the effect of low-dose diuretics on the serum cystatin $\mathrm{C}$ (CysC) and serum creatinine (Scr) levels, and on the prognosis in patients with asymptomatic chronic heart failure (HF). A total of 66 asymptomatic chronic HF patients were divided into the observation and control groups ( $\mathrm{n}=33$ in each group). Patients in the control group were treated with a routine treatment, while the patients in the observation group were treated with the diuretic hydrochlorothiazide along with the same routine treatment as the control group. The left ventricular ejection fraction (LVEF), serum CysC levels, Scr levels, heart function, prognosis, adverse reactions and complications of the patients in the two groups were compared prior to and following treatment. The LVEF increased in the two groups following treatment, while the levels of serum CysC and Scr decreased. The LVEF in the observation group increased following treatment for 1, 3 and 6 months compared with the LVEF values in the control group. In addition, the levels of serum $\mathrm{CysC}$ and Scr in the observation group were found to be lower compared with those in the control group $(\mathrm{P}<0.05)$. The incidence of adverse prognosis following treatment for 6 months in the observation group was lower compared with that in the control group $(\mathrm{P}<0.05)$. The proportion of HF patients with New York Heart Association (NYHA) class I and II increased following treatment for 6 months in the two groups $(\mathrm{P}<0.05)$. However, in the observation group, a higher number of patients exhibited class I and II disease after treatment for 6 months compared with the number in the control group $(\mathrm{P}<0.05)$. Furthermore, no statistically significant difference was observed between adverse reactions and complications in the two groups $(\mathrm{P}>0.05)$. In conclusion, low-dose diuretics may effectively improve the cardiac and renal functions and prognosis in asymptomatic chronic HF patients, without increasing the incidence of side effects.
\end{abstract}

Correspondence to: Dr Guanchang Cheng, Department of Cardiology, Henan University Huaihe Hospital, 115 Ximen Street, Kaifeng, Henan 475000, P.R. China

E-mail: guanchangchengcn@126.com

Key words: low dose, diuretics, asymptomatic, chronic heart failure, cystatin $\mathrm{C}$

\section{Introduction}

In recent years, environmental and lifestyle changes, as well as the increase in the aging population, have led to a subsequent increase in the incidence rates of different cardiovascular and cerebrovascular diseases (1). Chronic heart failure (HF) refers to the weakening of myocardial contractility, in which the normal cardiac output cannot be maintained due to chronic primary myocardial disease, long-term high blood pressure or circulatory overload (2). Chronic HF can involve the left side, right side or whole heart (3). Asymptomatic HF is a common disease and hospitalization cause in elderly patients (4), which does not present evident symptoms, but is the end-stage of various types of heart disease. In $\mathrm{HF}$, various body functions of the patients are significantly impaired and the immune function decreases. Adverse cardiac events frequently occur in patients with $\mathrm{HF}$, and may result in mortality. The survival rate of HF is low and the prognosis is poor (5). Therefore, the development of more effective medication is essential in order to improve the function of various organs and the prognosis of patients.

Diuretics are an important, indispensable part of the standard treatment for HF. These drugs can improve the clinical symptoms of patients with $\mathrm{HF}$, when combined with angiotensin-converting enzyme inhibitors, angiotensin receptor blockers and other drugs (6,7). However, diuretics may lead to various adverse reactions, affecting the therapeutic efficiency of the treatment. In addition, certain patients may refuse the use of diuretics during the treatment. In such cases, the recurrence rate of $\mathrm{HF}$ is high, with an increased number of re-hospitalized patients, while the treatment efficiency can be reduced and a longer course of treatment is required (8). Previous studies have shown that regular long-term use of low-dose diuretics can improve the cardiac function of patients. For instance, in the study by Cocco and Jerie (9), the left ventricular ejection fraction (LVEF) of patients increased following treatment with diuretics, while the side effects and complications decreased. Cystatin $\mathrm{C}(\mathrm{CysC})$ is a type of cysteine protease inhibitor. When the renal function declines, CysC levels increase much earlier than the levels of urea and creatinine. Therefore, the level of $\mathrm{CysC}$ can be used in the evaluation and early prediction of changes in the renal function $(10,11)$. A decrease in the heart function of patients has been shown to be accompanied by abnormal kidney function (12). Therefore, improving the cardiac function, renal function and prognosis of patients with 
HF is crucial. Only a limited number of studies have reported the effects of diuretics on the level of serum CysC and the prognosis of patients with HF (13).

The aim of the present study was to analyze changes in the LVEF and the levels of serum creatinine (Scr) and serum $\mathrm{Cys} C$ after long-term use of low-dose diuretics in patients with asymptomatic chronic HF. In addition, the study investigated the effects of long-term regular use of low-dose diuretics on the prognosis, cardiac function classification, occurrence of adverse reactions and complications in patients with asymptomatic chronic HF.

\section{Subjects and methods}

Subjects. A total of 66 patients with asymptomatic chronic HF who were admitted to the Henan University Huaihe Hospital (Kaifeng, China) between January 2012 and January 2014 were enrolled in the present study. The patients were randomly divided into the observation (17 men and 16 women; age range, 45-78 years; mean age, $63.52 \pm 8.47$ years) and control group (18 men and 15 women; age range, $42-76$ years; mean age, $63.15 \pm 8.82$ years), with 33 patients in each group. The study included patients who met the diagnostic criteria for HF (14) and provided informed consent. Patients with liver and/or kidney dysfunction, pregnant or lactating patients, and patients complicated by other severe cardiovascular diseases, cerebrovascular diseases or other causes of severe arrhythmia were excluded from the study. The heart function of patients was classified according to the New York Heart Association (NYHA) functional classification (15). This study was conducted in accordance with the declaration of Helsinki and with approval from the Ethics Committee of Henan University Huaihe Hospital. Written informed consent was obtained from all participants.

Treatment method. The patients were treated with angiotensin-converting enzyme inhibitors $(25 \mathrm{mg}$ Captopril; Wanchang Pharmaceutical Co., Ltd., Xi'an, China), $\beta$ blockers (25 mg Porpranolol; Baiyunshan Pharmaceutical Co. Ltd., Guangzhou, China) and Digitalis (0.5 mg Lanatoside; Fuxing Pharmaceutical Co., Ltd., Shanghai, China) in the control group, which is routine treatment for HF patients. Patients in the observation group were treated with the same treatment as the control group, in addition to $5-15 \mathrm{mg} /$ day of the diuretic hydrochlorothiazide (Shanxi Yunpeng Pharmaceutical Co., Ltd, Linfen, China; approval no. H14020796) for long-term treatment, along with the same routine treatment as the control group.

Detection method. In order to evaluate the effect of the diuretic treatment in HF patients, the LVEF, levels of Scr and serum CysC and NYHA cardiac functional classification were determined, while any adverse reactions and complications prior to and following the diuretic treatment were recorded in the two groups. The LVEF, serum CysC levels and Scr levels were detected in the patient serum at 1 day before the treatment, and at 1 week, 1 month, 3 months and 6 months after the treatment. A total of $5 \mathrm{ml}$ fasting blood was extracted from all participants, centrifuged at $4,000 \mathrm{xg}$ at $4^{\circ} \mathrm{C}$ for $10 \mathrm{~min}$ and stored at a constant temperature of $-20^{\circ} \mathrm{C}$.
Next, the level of serum CysC was detected using the latex enhanced turbidimetric method (16), while the level of Scr was detected using an enzymatic method (17). Scr detection kits were provided by Nanjing Sen Beijia Biological Technology Co., Ltd. (Nanjing, China). An ELx800 optical absorbance microplate reader (BioTek Instruments, Inc., Winooski, VT, USA) was used to measure the absorbance values at $570 \mathrm{~nm}$. LVEF was detected using the HP Sonos 2000 ultrasonic diagnostic apparatus (Hewlett-Packard, Palo Alto, CA, USA) and the ultrasonic probe frequency was 3.5-7 MHz. All procedures were performed in strict accordance with the manufacturer's instructions.

Evaluation method. NYHA functional classification (15) was determined according to the activity degree of symptoms of induced by HF. The degree of cardiac function damage is divided into the following four classes: Class I, in which the patient suffers from heart disease, but daily physical activity is not limited and does not cause excessive fatigue, palpitations, shortness of breath or angina pectoris; class II, in which the physical activity of patients with heart disease is mildly restricted, with no symptoms at rest, while general physical activity can cause excessive fatigue, palpitations, shortness of breath or angina pectoris; class III, in which heart disease causes significant restriction in physical activity, with no symptoms at rest, while less than ordinary physical activity can result in excessive fatigue, palpitations, shortness of breath or angina pectoris; and class IV, in which the patients with heart disease are unable to engage in any physical activity, while the symptoms of HF appear at rest and discomfort is aggravated subsequent to any physical activity.

Statistical analysis. All data were analyzed using SPSS version 17.0 software (SPSS Inc., Chicago, IL, USA). Quantitative data were compared using the $t$ test, while enumeration data were compared with the $\chi^{2}$ test. $\mathrm{P}<0.05$ was considered to indicate a statistically significant difference.

\section{Results}

NYHA classification of patients. In the observation group, there were 2 cases with NYHA class II, 23 cases with class III and 8 cases with class IV. In the control group, there were 3 cases with NYHA class II, 23 cases with class III and 7 cases with class IV. No statistically significant differences in the gender, age, NYHA cardiac functional classification were observed between the two groups $(\mathrm{P}>0.05)$.

Comparison of LVEF, serum CysC levels and Scr levels prior to and following treatment in the two groups. LVEF, serum CysC levels and Scr levels prior to diuretic treatment were compared between the two groups, and no statistically significant difference was observed ( $P>0.05$; Table I). In the observation group, LVEF after treatment for 1 week, 1 month, 3 months and 6 months increased compared with the values prior to treatment. In addition, the levels of serum CysC and Src in the observation group were found to be decreased at these time points compared with the levels prior to diuretic treatment. In the control group, LVEF increased after 
Table I. Comparison of LVEF, Scr levels and serum CysC levels prior to and following diuretic treatment in the two groups.

\begin{tabular}{|c|c|c|c|c|}
\hline Levels at each time point & Observation group & Control group & t test & P-value \\
\hline \multicolumn{5}{|l|}{ Prior to treatment } \\
\hline LVEF, \% & $25.45 \pm 4.92$ & $26.02 \pm 4.22$ & 0.25 & $>0.05$ \\
\hline CysC, mg/l & $6.42 \pm 2.14$ & $6.39 \pm 2.66$ & 0.18 & $>0.05$ \\
\hline $\mathrm{Scr}, \mu \mathrm{mol} / 1$ & $265.41 \pm 53.22$ & $259.25 \pm 51.98$ & 1.43 & $>0.05$ \\
\hline \multicolumn{5}{|l|}{ After treatment for 1 week } \\
\hline LVEF, \% & $35.22 \pm 3.64^{\mathrm{a}}$ & $31.45 \pm 3.65$ & 1.82 & $>0.05$ \\
\hline CysC, mg/l & $4.87 \pm 1.05^{\mathrm{a}}$ & $5.49 \pm 1.24$ & 2.26 & $>0.05$ \\
\hline $\mathrm{Scr}, \mu \mathrm{mol} / 1$ & $185.69 \pm 46.88^{\mathrm{a}}$ & $192.84 \pm 48.72$ & 2.17 & $>0.05$ \\
\hline \multicolumn{5}{|l|}{ After treatment for 1 month } \\
\hline LVEF, \% & $43.55 \pm 4.26^{\mathrm{a}}$ & $33.68 \pm 4.75^{b}$ & 2.62 & $<0.05$ \\
\hline CysC, mg/l & $3.26 \pm 0.59^{\mathrm{a}}$ & $4.18 \pm 0.88^{b}$ & 3.54 & $<0.05$ \\
\hline $\mathrm{Scr}, \mu \mathrm{mol} / \mathrm{l}$ & $125.78 \pm 32.55^{\mathrm{a}}$ & $146.25 \pm 36.74^{\mathrm{b}}$ & 3.89 & $<0.05$ \\
\hline \multicolumn{5}{|l|}{ After treatment for 3 months } \\
\hline LVEF, \% & $48.25 \pm 4.88^{\mathrm{a}}$ & $36.47 \pm 4.69^{b}$ & 3.45 & $<0.05$ \\
\hline CysC, mg/l & $2.24 \pm 0.48^{\mathrm{a}}$ & $3.96 \pm 0.72^{b}$ & 4.16 & $<0.05$ \\
\hline $\mathrm{Scr}, \mu \mathrm{mol} / 1$ & $98.41 \pm 21.59^{\mathrm{a}}$ & $123.55 \pm 23.72^{b}$ & 4.87 & $<0.05$ \\
\hline \multicolumn{5}{|l|}{ After treatment for 6 months } \\
\hline LVEF, \% & $50.86 \pm 5.22^{\mathrm{a}}$ & $38.22 \pm 5.12^{\mathrm{b}}$ & 4.28 & $<0.05$ \\
\hline CysC, mg/l & $1.65 \pm 0.26^{\mathrm{a}}$ & $2.84 \pm 0.53^{\mathrm{b}}$ & 5.27 & $<0.05$ \\
\hline $\mathrm{Scr}, \mu \mathrm{mol} / 1$ & $78.52 \pm 13.78^{\mathrm{a}}$ & $106.65 \pm 17.43^{\mathrm{b}}$ & 5.93 & $<0.05$ \\
\hline
\end{tabular}

${ }^{\mathrm{a}} \mathrm{P}<0.05$, vs. observation group before treatment; ${ }^{\mathrm{b}} \mathrm{P}<0.05$, vs. control group before treatment. LVEF, left ventricular ejection fraction; Scr, serum creatinine; CysC, cystatin $\mathrm{C}$.

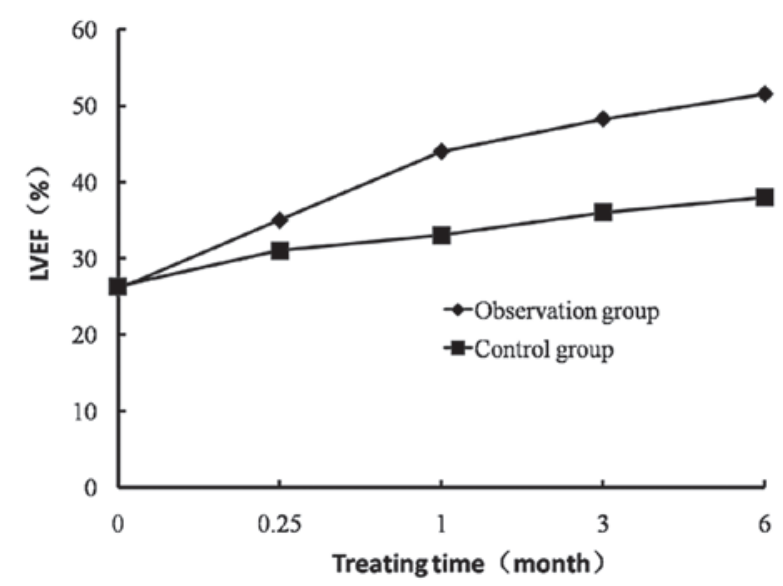

Figure 1. LVEF levels at different treatment time points in the two groups. LVEF, left ventricular ejection fraction.

treatment for 1, 3 and 6 months compared with the value prior to treatment, whereas the levels of serum CysC and Scr decreased at these time points compared to the levels prior to treatment. Furthermore, LVEF values in the observation group after treatment for 1, 3 and 6 months was higher compared with those in the control group $(\mathrm{P}<0.05)$. The levels of serum $\mathrm{CysC}$ and $\mathrm{Scr}$ in the observation group were lower at the aforementioned time points compared with the levels in the control group, and the difference was statistically significant $(\mathrm{P}<0.05$; Table I; Figs. 1-3).

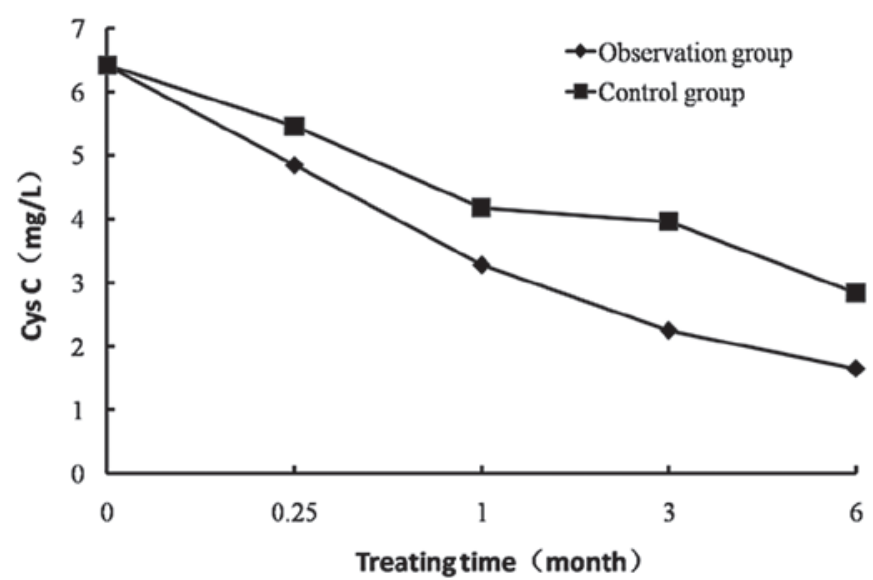

Figure 2. CysC levels at different treatment time points in the two groups. CysC, cystatin C.

Comparison of NYHA class prior to and following diuretic treatment in the two groups. The number of patients in each NYHA class prior to treatment was not significantly different between the two groups ( $\mathrm{P}>0.05$; Table IIA). The number of patients with class I and II heart function in the two groups increased following treatment, while the number of patients with class III and IV cardiac function decreased. In the observation group, the number of patients with cardiac function of class I and II increased following treatment, whereas the number of patients with cardiac function of class III and IV decreased following 
Table II. Comparison of NYHA cardiac function classification prior to and following treatment in the two groups (n, \%).

A, Prior to treatment

\begin{tabular}{|c|c|c|c|c|}
\hline Parameter & Observation group & Control group & $\chi^{2}$ test & P-value \\
\hline Number of patients, $n$ & 33 & 33 & - & - \\
\hline \multicolumn{5}{|c|}{ NYHA classification, n (\%) } \\
\hline Class I & $0(0.00)$ & $0(0.00)$ & - & - \\
\hline Class II & $2(6.06)$ & $3(9.09)$ & 3.24 & $>0.05$ \\
\hline Class III & $23(69.69)$ & $23(69.69)$ & - & - \\
\hline Class IV & $8(24.24)$ & $7(21.21)$ & 1.42 & $>0.05$ \\
\hline
\end{tabular}

B, After treatment for 6 months

\begin{tabular}{|c|c|c|c|c|}
\hline Parameter & Observation group & Control group & $\chi^{2}$ test & P-value \\
\hline Number of patients, $n$ & 32 & 31 & - & - \\
\hline \multicolumn{5}{|c|}{ NYHA classification, n (\%) } \\
\hline Class I & $3(9.38)^{\mathrm{a}}$ & $1(3.23)^{b}$ & 6.62 & $<0.05$ \\
\hline Class II & $9(28.13)^{\mathrm{a}}$ & $4(12.90)^{b}$ & 7.84 & $<0.01$ \\
\hline Class III & $18(56.25)^{\mathrm{a}}$ & $22(70.97)^{b}$ & 4.66 & $<0.05$ \\
\hline Class IV & $2(6.25)^{\mathrm{a}}$ & $4(12.90)^{\mathrm{b}}$ & 5.97 & $<0.05$ \\
\hline
\end{tabular}

${ }^{\text {a }} \mathrm{P}<0.05$, vs. observation group before treatment; ${ }^{\mathrm{b}} \mathrm{P}<0.05$, vs. control group before treatment. Lack of significant difference is indicated by a hyphen (-). NYHA, New York Heart Association.

Table III. Comparison of prognosis after treatment for 6 months in the two groups ( $\mathrm{n}=33$ each group).

\begin{tabular}{|c|c|c|c|c|}
\hline Prognosis & Observation group & Control group & $\chi^{2}$ test & P-value \\
\hline Improvement, n (\%) & $26(78.79)$ & $20(60.61)$ & 4.82 & $<0.05$ \\
\hline Deterioration, n (\%) & $6(18.18)$ & $11(33.33)$ & 6.48 & $<0.05$ \\
\hline Mortality, n (\%) & $1(3.03)$ & $2(6.06)$ & 6.25 & $<0.05$ \\
\hline Incidence of adverse prognosis, $\%$ & 21.21 & 39.39 & 4.82 & $<0.05$ \\
\hline
\end{tabular}

treatment, when compared with that in the control group, and the differences were statistically significant $(\mathrm{P}<0.05$; Table IIB).

Comparison of prognosis in the two groups. In the observation group, the number of patients with improved prognosis increased after treatment for 6 months compared with the control group (Table III). In addition, the number of HF patients with deteriorated prognosis, mortality or incidence of adverse prognosis were significantly lower in the observation group, compared with those in the control group following treatment for 6 months $(\mathrm{P}<0.05$; Table III). Thus, the results indicated that treatment with low-dose diuretics improved the prognosis of patients with HF.

Comparison of adverse reactions and complications. The incidence of headaches, dizziness, anemia, allergies, difficulty in breathing, hearing loss, and other adverse reactions and complications was compared between the two groups, with no statistically significant differences observed ( $P>0.05$; Table IV).

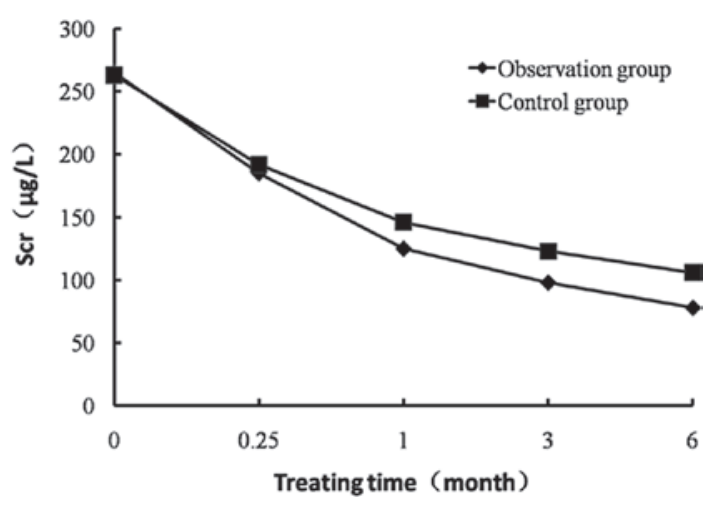

Figure 3. Comparison of the levels of Scr at different treatment times in the two groups. Scr, serum creatinine.

\section{Discussion}

HF is frequently associated with asymptomatic left ventricular dysfunction (18). Patients normally present left ventricular 
Table IV. Comparison of adverse reactions and complications in the two groups ( $\mathrm{n}=33$ each group).

\begin{tabular}{lcc}
\hline Parameter & Observation group & Control group \\
\hline Headache and dizziness, $\mathrm{n}$ & 1 & 1 \\
Anemia, $\mathrm{n}$ & 1 & 1 \\
Allergies, $\mathrm{n}$ & 2 & 1 \\
Difficulty in breathing, $\mathrm{n}$ & 0 & 1 \\
Hearing loss, $\mathrm{n}$ & 15.15 & 0 \\
Incidences of adverse reactions & & 12.12 \\
and complications, $\%^{\mathrm{a}}$ &
\end{tabular}

${ }^{\mathrm{a}}$ Comparison of incidence of adverse reactions and complications between the two groups resulted in $\chi^{2}$ test $=2.43$ and $\mathrm{P}>0.05$.

systolic dysfunction without evident symptoms of congestive HF. Asymptomatic HF often occurs in the elderly population, severely affecting the patient's health and quality of life, and may be life-threatening $(19,20)$. The incidence of HF in patients with cerebral vascular disease is high, while cardiovascular and cerebral amplification effects may result in alterations in the cardiac function of patients. In the early stages of the disease, the patients often present no evident symptoms. With time, the disease may develop or worsen into dominant HF, which may lead to the occurrence of an adverse prognosis $(21,22)$; therefore, it is important to detect, prevent and timely treat chronic HF at an early stage.

Diuretics are commonly administered for the treatment of $\mathrm{HF}$, since they can reduce the occurrence of sodium and water retention through inhibition of tubular reabsorption of sodium and chloride, thereby reducing the cardiac load in order to improve heart function (23). Asymptomatic chronic HF often develops into HF with evident symptoms, having severe and even life-threatening effects on the health of patients and resulting in the incidence of adverse prognosis. Therefore, the timely and effective treatment of HF patients is crucial. Serum CysC levels are altered with changes in renal function, and thus can be used as an index for the early detection of such changes (24). There are few studies reporting the effect of low-dose diuretics on renal function and prognosis $(13,25)$. Determination of the effect of long-term regular use of low-dose diuretics on cardiac function, renal function and prognosis of patients with asymptomatic HF may provide a strong clinical basis for the improvement of prevention and prognosis, avoiding organ damage.

The results of the present study showed that the LVEF and heart function of patients with asymptomatic HF were significantly reduced compared with normal levels, which was consistent with the results of Parry et al (26). In addition, the levels of Scr, CysC and other markers of renal function in patients with asymptomatic chronic HF were significantly increased compared with normal levels in the present study, suggesting that the renal function was evidently damaged. Asymptomatic HF involves multiple systemic organs prior to the appearance of evident symptoms, and thus its early diagnosis and treatment are crucial. The mechanism of abnormal cardiac and renal function in patients with asymptomatic HF may be explained as follows: HF leads to long-term overload compensatory work, which may cause significant cardiac damage, reduce the heart function of patients and result in the reduction of LVEF. Abnormalities in the heart function frequently lead to the alteration of hemodynamics, affecting renal blood circulation, which can result in the decline of renal function and the increase of Scr and CysC levels (27). In the present study, following treatment with long-term regular low-dose diuretics, the LVEF of the patients with asymptomatic HF increased consistently, while the levels of Scr and CysC continuously declined throughout the treatment. The improvement in the heart and renal function of the patients indicated that long-term regular low-dose diuretic-based treatment was beneficial, improving the organ function in patients with chronic HF.

The underlying mechanism through which long-term regular use of low-dose diuretics elevate LVEF and decrease the levels of $\mathrm{Scr}$ and serum CysC may be as follows: A low dose of diuretics can have a long-term positive effect on the kidneys, inhibit the overactivation of the rennin-angiotensin-aldosterone system in patients with asymptomatic HF, and alleviate the symptoms of HF. Furthermore, low-dose diuretic treatment effectively inhibits the reabsorption of sodium and chloride in the glomerulus in order to decrease their levels in the body, while it also reduces the occurrence of sodium and water retention, reduces the cardiac preload in order to improve heart function, and improves the LVEF. The improvement of cardiac function can also reduce, to a certain extent, the effects of HF on hemodynamics, improving the renal circulation and function, and reducing the levels of Scr, CysC and other associated indexes of renal function (28). Following treatment with long-term low-dose diuretics, a higher number of patients with asymptomatic HF exhibited improved HF-related symptoms compared with the control patients, while the number of patients with worsened disease or mortality due to renal failure reduced. In addition, the heart function classification of patients increased and the prognosis was evidently improved, following diuretic treatment. Furthermore, the incidence of adverse reactions and complications (including headache, dizziness, anemia, allergies, breathing difficulties and hearing loss) did not increase in patients treated with diuretics, compared with the control patients. Due to the short observation time in the present study, the interval between heart and renal function detection was longer, while a large number of factors affected the heart function, renal function and prognosis. Therefore, the effect of 
low-dose diuretics on patients with asymptomatic chronic HF should be investigated further in an in-depth study with longer observation time.

In conclusion, long-term low-dose diuretic treatment effectively improved the heart function, renal function and prognosis in patients with asymptomatic chronic HF. Furthermore, this treatment did not increase the occurrence of adverse reactions and complications. Thus, administration of low-dose diuretics is an effective treatment for asymptomatic chronic HF.

\section{References}

1. Orth-Gomér K: Psychosocial and behavioral aspects of cardiovascular disease prevention in men and women. Curr Opin Psychiatry 20: 147-151, 2007

2. Berezin AE, Kremzer AA, Samura TA and Martovitskaya YV: Circulating endothelial-derived apoptotic microparticles in the patients with ischemic symptomatic chronic heart failure: Relevance of pro-inflammatory activation and outcomes. Int Cardiovasc Res J 8: 116-123, 2014

3. Maughan D and Toth M: Discerning primary and secondary factors responsible for clinical fatigue in multisystem diseases. Biology (Basel) 3: 606-622, 2014.

4. Lang NN, Wong CM, Dalzell JR, Jansz S, Leslie SJ and Gardner RS: The ease of use and reproducibility of the Alere ${ }^{\mathrm{TM}}$ Heart Check System: A comparison of patient and healthcare professional measurement of BNP. Biomark Med 8: 791-796, 2014

5. Armstrong C: ACCF and AHA release guidelines on the management of heart failure. Am Fam Physician 90: 186-189, 2014

6. Nativi-Nicolau J, Ryan JJ and Fang JC: Current therapeutic approach in heart failure with preserved ejection fraction. Heart Fail Clin 10: 525-538, 2014.

7. Imamura T, Kinugawa K, Ohtani T, Sakata Y,Higo T, Kinugawa S, Tsutsui H, Sunagawa K and Komuro I: Assessment of quality of life during long-term treatment of tolvaptan in refractory heart failure: Design and rationale of the AQUA-TLV study. Int Heart J 55: 264-267, 2014

8. Rad EM and Assadi F: Management of hypertension in children with cardiovascular disease and heart failure. Int J Prev Med 5 (Suppl 1): S10-S16, 2014.

9. Cocco G and Jerie P: Comparison between ivabradine and low-dose digoxin in the therapy of diastolic heart failure with preserved left ventricular systolic function. Clin Pract 3: e29, 2013.

10. Pitt B, Pfeffer MA, Assmann SF, Boineau R, Anand IS, Claggett B, Clausell N, Desai AS, Diaz R, Fleg JL, et al: Spironolactone for heart failure with preserved ejection fraction. N Engl J Med 370: 1383-1392, 2014.

11. De Vecchis R, Esposito C and Ariano C: Efficacy and safety assessment of isolated ultrafiltration compared to intravenous diuretics for acutely decompensated heart failure: A systematic review with meta-analysis. Minerva Cardioangiol 62: 131-146, 2014.

12. Mpe MT, Klug EQ, Silwa KS, Hitzeroth J and Smith DA: Heart failure society of South Africa (HeFSSA) perspective on the European society of cardiology (ESC) 2012 chronic heart failure guideline. S Afr Med J 103 (9 Suppl 2): 660-667, 2013.
13. Verbrugge FH, Nijst P, Dupont M, Reynders C, Penders J, Tang WH and Mullens W: Prognostic value of glomerular filtration changes versus natriuretic response in decompensated heart failure with reduced ejection. J Card Fail 20: 817-824, 2014.

14. Seronde MF, Laribi S, Collins SP, Deye N, Logeart D, Plaisance P, Cohen-Solal A and Mebazaa A: Heart failure diagnosis in acute conditions has high agreement with inpatient diagnosis. Eur J Emerg Med: Feb 23, 2015 (Epub ahead of print).

15. Miller-Davis C, Marden S and Leidy NK: The New York Heart Association Classes and functional status: What are we really measuring? Heart Lung 35: 217-224, 2006.

16. Uemura O, Ushijima K, Nagai T, Yamada T, Hayakawa H, Nabeta Y, Shinkai Y, Koike K and Kuwabara M: Reference serum cystatin C levels in Japanese children. Clin Exp Nephrol 14: 453-456, 2010.

17. Wang X, Xu G, Li H, Liu Y and Wang F: Reference intervals for serum creatinine with enzymatic assay and evaluation of four equations to estimate glomerular filtration rate in a healthy Chinese adult population. Clin Chim Acta 412: 1793-1797, 2011.

18. Moukarbel GV and Solomon SD: Treatment of asymptomatic left ventricular dysfuntion. Curr Treat Options Cardiovasc Med 10: 476-485, 2008.

19. Rami K: Aggressive salt and water restriction in acutely decompensated heart failure: is it worth its weight in salt? Expert Rev Cardiovasc Ther 11: 1125-1128, 2013.

20. Lee KK, Shilane D, Hlatky MA, Yang J, Steimle AE and Go AS: Effectiveness and safety of spironolactone for systolic heart failure. Am J Cardiol 112: 1427-1432, 2013.

21. Hasenfuß G, Edelmann F and Wachter R: Recommendations for the treatment of heart failure: What's new? Internist (Berl) 54: 1141-1151, 2013 (In German).

22. Ferreira JP, Santos M, Almeida S, Marques I, Bettencourt P and Carvalho $\mathrm{H}$ : Tailoring diuretic therapy in acute heart failure: Insight into early diuretic response predictors. Clin Res Cardiol 102: 745-753, 2013.

23. Vaduganathan M, Marti CN, Georgiopoulou VV, Kalogeropoulos AP and Butler J: Classification of patients hospitalized for heart failure. Heart Fail Clin 9: 277-283, 2013.

24. Frankenstein L, Katus HA, Grundtvig M, Hole T, de Blois J, Schellberg D, Atar D, Zugck C and Agewall S; Norwegian Heart Failure Registry steering committee: Association between spironolactone added to beta-blockers and ACE inhibition and survival in heart failure patients with reduced ejection fraction: A propensity score-matched cohort study. Eur J Clin Pharmacol 69: $1747-1755,2013$.

25. Ng TM, Hshieh S, Chan CY and Elkayam U: Clinical experience with low-dose continuous infusion of furosemide in acute heart failure: Assessment of efficacy and safety. J Cardiovasc Pharmacol Ther 17: 373-381, 2012.

26. Parry HM, Doney AS, Palmer CN and Lang CC: State of play of pharmacogenetics and personalized medicine in heart failure. Cardiovasc Ther 31: 315-322, 2013.

27. O'Dell KM, Kalus JS, Kucukarslan S and Czerska B: Nesiritide for secondary pulmonary hypertension in patients with end-stage heart failure. Am J Health Syst Pharm 62: 606-609, 2005.

28. Chen HH, Martin FL, Gibbons RJ, Schirger JA, Wright RS, Schears RM, Redfield MM, Simari RD, Lerman A, Cataliotti A and Burnett JC Jr: Low-dose nesiritide in human anterior myocardial infarction suppresses aldosterone and preserves ventricular function and structure: A proof of concept study. Heart 95: 1315-1319, 2009. 